\title{
Study on Fleet Assignment Problem Model and Algorithm
}

\author{
Yaohua Li and Na Tan \\ Aeronautical Engineering College, Civil Aviation University of China, Tianjin 300300, China \\ Correspondence should be addressed to Yaohua Li; li_yaohua@sina.com
}

Received 9 January 2013; Accepted 26 February 2013

Academic Editor: Jun Zhao

Copyright (C) 2013 Y. Li and N. Tan. This is an open access article distributed under the Creative Commons Attribution License, which permits unrestricted use, distribution, and reproduction in any medium, provided the original work is properly cited.

\begin{abstract}
The Fleet Assignment Problem (FAP) of aircraft scheduling in airlines is studied, and the optimization model of FAP is proposed. The objective function of this model is revenue maximization, and it considers comprehensively the difference of scheduled flights and aircraft models in flight areas and mean passenger flows. In order to solve the model, a self-adapting genetic algorithm is supposed to solve the model, which uses natural number coding, adjusts dynamically crossover and mutation operator probability, and adopts intelligent heuristic adjusting to quicken optimization pace. The simulation with production data of an airline shows that the model and algorithms suggested in this paper are feasible and have a good application value.
\end{abstract}

\section{Introduction}

Fleet Assignment Problem (FAP) is to assign an aircraft model for each scheduled flight according to the capability of passengers, running cost, and planned revenue of each fleet. This is an important work of aircraft scheduling and planning in airlines. The results of FAP affect not only the cost and revenue of airlines, but also the continuing works, such as linking problem between flights, aircraft's maintenance route, crew assigning, and flight gate assigning. The aircraft scheduling is a controlling work of production scheduling in airlines. Because of the importance and complexity of the aircraft scheduling work in the air transport, the in-depth research and application have been carried out in aviation developed countries of Europe and America [1-3]. In China, because airlines had small amount of aircrafts a few years ago, they have not paid more attention to production plan and management and their planning mode was simple and manned. At the same time, the research on civil aviation production planning management is few. Recently, with the number of airlines' aircrafts increasing, the aviation transport market opening, and the aviation market competition pricking up, airlines wake up to the importance and urgency of production scheduling and planning management gradually. But in general, the theory research on aircraft planning and scheduling is still in the underway phase. Reference [4] presents the notion of flight purity and builds fleet assignment model subjected to flight purity according to the characters of Chinese airline network and flight scheduling. A robust mathematical model for the fleet scheduling problem is put forward [5]. According to the data provided by an airline, the computational experiment performed with the improved Grover's algorithm shows the effectiveness of the proposed model and improves the robustness of the decision. The aircraft scheduling problem based on cooperative multitask assignment is studied [6], and the approach applies branch-and-price algorithm to the cost optimization model with maintenance constraints, and mathematical model of daily utilization ratio is established. A model of flight-string VRP based on the time unit of week is suggested [7], and a parthenogenetic algorithm is suggested for solving the model. In China, although some scholars have studied the problem of aircraft planning and scheduling, the plan of aircraft scheduling in airlines is completed with manpower or half-manpower mode. The level of automation is not tall, and the information system based on the mature models and algorithms is few.

In this paper, according to Chinese factual situation, FAP in airlines is studied in order to establish a foundation for aircraft planning and scheduling automation. Based on the research result, an optimization model of FAP is proposed, which takes the total revenue maximum as objective and can assign an appropriate aircraft type to each flight. For solving the complicated optimization model, an improved genetic algorithm is suggested, which can find out optimal solution 
quickly. After deep studying, the model and algorithm can be applied in production scheduling of other countries' airlines.

\section{FAP Optimization Model}

2.1. Problem Description. In production scheduling of airlines, Fleet Assignment Planning is to assign the most appropriate aircraft type to each flight. The flying performance of different aircraft model is different, for example, voyage range, flying altitude ceiling, maximum take-off weight, and climbing ability. So, a particular route is not suitable for all models of the aircraft to perform. In addition, different models have different seating layout, and their operating costs are not the same. For instance, the seats number of the B737-300 aircraft is about 144, and its direct operating costs are between 30 and 50 thousands of RMB per hour. But the A340-200 aircraft can seat up to about 380 people, and its direct operating cost is more than 100,000 of RMB per hour. The basis for the development of the work is the airworthiness limitations of flight route on aircraft models, each model's cabin distribution, operational cost analysis of the models in the different routes, as well as forecasts of passenger and freight traffic on each flight. The goal is to optimize the allocation of models to flight, in order to minimize the operating costs to complete the flight running tasks.

2.2. FAP Optimization Model Building. Considering the feature of Chinese flight route net and flight plan, under constraints of determined flight schedules, not considering the flight stopovers, only considering aircraft A check, and enough airport capacity, the FAP model which considers the models match, model flying area, as well as the traffic match conditions is proposed as follows:

$$
\begin{aligned}
& \quad \max \sum_{i=1}^{m} \sum_{j=1}^{n} c_{i j} x_{i j}, \\
& \text { s.t. } \\
& \quad \sum_{i=1}^{m} \sum_{j=1}^{n} x_{i j}=m, \\
& \quad \sum_{j=1}^{n} x_{i j}=1, \\
& x_{i j} r_{j} \geqslant R_{i}, \quad i=1,2, \ldots, m, j=1,2, \ldots, n, \\
& x_{i j} p_{j} \geqslant P_{i}, \quad i=1,2, \ldots, m, j=1,2, \ldots, n, \\
& x_{i j} d_{j} \geqslant D_{i}, \quad i=1,2, \ldots, m, j=1,2, \ldots, n,
\end{aligned}
$$

where

$$
\begin{aligned}
& i=1,2, \ldots, m ; m \text { is overall flight number, } \\
& j=1,2, \ldots, n ; n \text { is the number of aircraft models, } \\
& c_{i j} \text {; the revenue of aircraft model } j \text { to perform the } \\
& \text { flight } i,
\end{aligned}
$$

$c_{i j}=R_{i j}-\mathrm{OC}_{i j}^{f}-\mathrm{OC}_{i j}^{a} ; R_{i j}, \mathrm{OC}_{i j}^{f}$, and $\mathrm{OC}_{i j}^{a}$ are the revenue, fixed operating costs, and the variable operating cost of model $j$ to fly flight $i$,

$x_{i j}= \begin{cases}1, & \text { flight } i \text { is performed by the model } j, \\ 0, & \text { or else, }\end{cases}$

$r_{j}$, the suitable flying area code of model $j$,

$R_{i}$, the minimum flying area code required by flight $i$,

$p_{j}$, the passenger capacity of model $j$,

$P_{i}$, the average traffic of flight $i$,

$d_{j}$, model code of the model $j$,

$D_{i}$, the model code required by flight $i$.

The objective function (1) means that the total income of all flights is largest, after the aircraft types are assigned to all flights considering the bulk of the flights of global optimization. Constraint (2) is to ensure that an equal number of models are selected for flights. Constraint (3) is to ensure that only one model is assigned to each flight. Constraint (4) is to ensure that the model assigned to the flight meets the flight area requirements. Constraint (5) is to ensure that the model assigned to the flight meets the flight traffic requirement. Constraint (6) is to ensure that the code of the model assigned to the flight is greater than the flight code. In order to calculate conveniently, the models flying area code and model code use a natural number coding according to difference of the actual situation; for example, 1, 2 represent the fly zone, representing the flight area of plains and plateaus. The model codes of 1, 2, 3 represent, respectively, the B737$300, \mathrm{~B} 737-800, \mathrm{~B} 757$, and so forth. Thus, in order to ensure the operational feasibility in the model calculations, all of these codings take the downward compatible form. That is, highgrade aircrafts can perform the flight requiring low-grade aircraft model, not the contrary.

\section{The Solving Algorithm of FAP Optimization Model}

It is difficult to solve the FAP optimization model with mathematical programming methods, because FAP is an NP-hard problem. The genetic algorithm (GA) is an adaptive search algorithm which is based on the natural evolution and selection mechanism. And it has been successfully applied to a variety of optimization problems. In this paper, an improved hybrid heuristic genetic algorithm is constructed to solve the model, considering the limitations of the general genetic algorithm. The algorithm uses natural number coding method and dynamically adjusts the crossover and mutation probability.

3.1. Intelligent Heuristic Adjustment for Infeasible Solutions. The usual method for solving constrained optimization problems is to convert it to unconstrained optimization problem, which incorporated the constrained constraints into the evaluation function using the method of weighting coefficients. 
TABLE 1: The information of aircraft models.

\begin{tabular}{lccc}
\hline $\begin{array}{l}\text { Aircraft } \\
\text { model code }\end{array}$ & Model & $\begin{array}{c}\text { Maximum } \\
\text { passenger } \\
\text { capacity }\end{array}$ & $\begin{array}{c}\text { Code of } \\
\text { flying area }\end{array}$ \\
\hline 1 & B737-800 & 170 & 1 \\
2 & A320 & 180 & 2 \\
3 & B757 & 239 & 2 \\
4 & A340 & 295 & 3 \\
\hline
\end{tabular}

Thus, although constrained optimization problems can be solved, infeasible solutions may exist in aviation production scheduling production. In order to guarantee that individuals of each generation are feasible solutions, the algorithm will filter the infeasible solution in each generation solutions for every individual and then adjust the infeasible solutions with intelligent heuristic adjustment method. The heuristic rules of the intelligent heuristic adjustment method are based on expert knowledge and relevant constraints. When the individual does not meet the constraint needed to be adjusted, the algorithm adjusts it according to the individual situation and determines the direction and size of adjustment with the expert knowledge rules. Its goal is to ensure that the adjusted individual is feasible solution and is adjusted along the optimized search direction.

\subsection{Dynamic Adjustment of the Crossover Probability $P_{c}$ and} Mutation Probability $P_{m}$. In order to avoid genetic algorithm falling into a local optimum value and having rapid convergence, genetic operator probability adjustment method in the algorithm is used to dynamically adjust the crossover and mutation probability after [8] is studied. In this paper, the crossover probability $P_{c}$ and mutation probability $P_{m}$ of each generation groups are dynamically adjusted according to the degree of concentration of the fitness value. The adjustment method is to establish a judgment standard with the maximum fitness value $f_{\max }$, minimum fitness value $f_{\min }$, and average fitness value $f_{\text {ave }}$. Generally, the initial crossover probability is set as $P_{c 1}=0.9, P_{c 2}=0.6$, and mutation probability is set as $P_{m 1}=0.1, P_{m 2}=0.001$. Thus, $P_{c}$ and $P_{m}$ are changed with the evaluation (fitness function) of solutions. When the solution has good performance, let $P_{c}$ and $P_{m}$ be small to help the algorithm's rapid convergence. When the solution is lower than the average fitness value, let $P_{c}$ and $P_{m}$ be high to prevent the algorithm from optimal solutions into local solution. The adjustment formulas are as follows:

$$
\begin{gathered}
P_{c}= \begin{cases}P_{c 1}-\left(P_{c 1}-P_{c 2}\right) \frac{f-f_{\mathrm{ave}}}{f_{\max }-f_{\mathrm{ave}}}, & f \geq f_{\mathrm{ave}}, \\
P_{c 1}, & f<f_{\mathrm{ave}},\end{cases} \\
P_{m}= \begin{cases}P_{m 1}-\left(P_{m 1}-P_{m 2}\right) \frac{f-f_{\text {ave }}}{f_{\mathrm{max}}-f_{\text {ave }},} & f \geq f_{\text {ave }}, \\
P_{m 1}, & f<f_{\text {ave }} .\end{cases}
\end{gathered}
$$

\subsection{Steps of the Improved Genetic Algorithm}

(1) Inputting the data required by model solving: read the corresponding data information to be calculated.

(2) Algorithm parameters initialization: determine the algorithm population numbers and the end of the maximum cycle algebra, the initial values of crossover probabilities $\left(P_{c 1}, P_{c 2}\right)$ and mutation probabilities $\left(P_{m 1}, P_{m 2}\right)$ are set. Then, initial generation chromosomes are given as the current generation chromosome based on the population numbers given.

(3) Heuristic correction of the current generation of chromosomes: check the infeasible solutions in chromosomes, and then correct infeasible solutions using the intelligent heuristic rules until they become the feasible solutions.

(4) Calculate the adaptation function value of the current generation of chromosome, and record the best individual as the optimal solution. Then, judge whether to satisfy the end criterion; if the answer is yes, jump to (8), or else, jump to (5).

(5) Adaptive dynamics: adjust the current chromosome probability, and calculate the probability of crossover and mutation $P_{c}, P_{m}$.

(6) Current chromosome genetic manipulation: Cross is completed with probability $P_{c}$, mutation operating is done with probability $P_{m}$, and then selecting operation is completed, which selects the best chromosome in the current generation.

(7) Generation of chromosomes will be selected as the current generation of chromosome; return to (3).

(8) Output current optimal solution as the solution of the algorithm.

\section{Simulation Research}

In order to validate the model and algorithm supposed in this paper, the data of a medium-sized airline, including 4 aircraft models, 50 flights, is selected to study. Raw data are shown in Tables 1, 2, and 3. In this paper, the basic genetic algorithm (GA) and improved adaptive genetic algorithm (IGA) have been used for a comparative study in order to show that the algorithm suggested in this paper is better.

The parameters are selected as follows: the number of population genetic algorithm is 20; the IGA initial crossover probabilities are $P_{c 1}=0.9, P_{c 2}=0.6$; mutation initial probabilities are $P_{m 1}=0.1 ; P_{m 2}=0.001$; the algorithm terminates criteria for successive iterations 1000 generation. Basic genetic algorithm (GA) parameters are selected as follows: the number of population genetic algorithm is 20; crossover probability is set as $P_{c}=0.90$, mutation probability is set as $P_{m}=0.10$, the algorithm terminates criteria for successive iterations 1000 generation. The simulation calculating result is given in Figure 1 and Table 2, the best values being 567.7 and 526.4, respectively. Basic genetic algorithm can find out a feasible solution, but the result is bad. The computational 
TABLE 2: The information of flights.

\begin{tabular}{|c|c|c|c|c|c|c|c|c|c|c|}
\hline $\begin{array}{l}\text { Sequence } \\
\text { number }\end{array}$ & $\begin{array}{l}\text { Flight } \\
\text { number }\end{array}$ & $\begin{array}{c}\text { Day in } \\
\text { week }\end{array}$ & Departure & $\begin{array}{l}\text { Time of } \\
\text { departure }\end{array}$ & Destination & Arrival time & $\begin{array}{l}\text { Model } \\
\text { code }\end{array}$ & $\begin{array}{c}\text { Mean } \\
\text { passengers }\end{array}$ & $\begin{array}{l}\text { Code of } \\
\text { flying area }\end{array}$ & $\begin{array}{l}\text { The result of } \\
\text { FAP model } \\
\text { and algorithm }\end{array}$ \\
\hline 1 & $\mathrm{nx} 001$ & 1 & PEK & $16: 10$ & MFM & $19: 35$ & 1 & 150 & 1 & 4 \\
\hline 2 & $\mathrm{nx} 001$ & 2 & PEK & $16: 10$ & MFM & $19: 35$ & 1 & 150 & 1 & 2 \\
\hline 3 & $\mathrm{nx} 001$ & 3 & PEK & $16: 10$ & MFM & 19:35 & 1 & 150 & 1 & 2 \\
\hline 4 & $\mathrm{nx} 001$ & 4 & PEK & $16: 10$ & MFM & $19: 35$ & 1 & 150 & 1 & 1 \\
\hline 5 & $\mathrm{nx} 001$ & 5 & PEK & $16: 10$ & MFM & $19: 35$ & 1 & 150 & 2 & 3 \\
\hline$\vdots$ & $\vdots$ & $\vdots$ & $\vdots$ & $\vdots$ & $\vdots$ & $\vdots$ & $\vdots$ & $\vdots$ & $\vdots$ & $\vdots$ \\
\hline 44 & nx197 & 3 & CTU & $17: 20$ & MFM & $19: 30$ & 3 & 150 & 1 & 3 \\
\hline 45 & nx197 & 5 & CTU & $17: 20$ & MFM & $19: 30$ & 3 & 200 & 1 & 2 \\
\hline 46 & nx197 & 7 & CTU & $17: 20$ & MFM & $19: 30$ & 3 & 200 & 1 & 3 \\
\hline 47 & nx198 & 3 & MFM & $14: 00$ & CTU & $16: 30$ & 3 & 150 & 3 & 2 \\
\hline 48 & nx198 & 1 & MFM & $14: 00$ & CTU & $16: 30$ & 3 & 150 & 3 & 1 \\
\hline 49 & nx198 & 5 & MFM & $14: 00$ & CTU & $16: 30$ & 3 & 200 & 3 & 2 \\
\hline 50 & nx198 & 7 & MFM & $14: 00$ & CTU & $16: 30$ & 3 & 200 & 3 & 1 \\
\hline
\end{tabular}

TABLE 3: The income and cost statistical data of models perform flights (money unit: ten thousands RMB).

\begin{tabular}{|c|c|c|c|c|c|c|c|c|c|c|c|c|}
\hline \multirow{4}{*}{$\begin{array}{l}\text { Flight } \\
\text { sequence }\end{array}$} & \multicolumn{12}{|c|}{ Model code } \\
\hline & \multicolumn{3}{|c|}{1} & \multicolumn{3}{|c|}{2} & \multicolumn{4}{|c|}{3} & \multirow{2}{*}{\multicolumn{2}{|c|}{4}} \\
\hline & \multicolumn{10}{|c|}{ Cost } & & \\
\hline & Revenue & $\begin{array}{c}\text { Fixed } \\
\text { cost }\end{array}$ & $\begin{array}{c}\text { Variable } \\
\text { cost }\end{array}$ & Revenue & $\begin{array}{c}\text { Fixed } \\
\text { cost }\end{array}$ & $\begin{array}{c}\text { Variable } \\
\text { cost }\end{array}$ & Revenue & $\begin{array}{c}\text { Fixed } \\
\text { cost }\end{array}$ & $\begin{array}{c}\text { Variable } \\
\text { cost }\end{array}$ & Revenue & $\begin{array}{c}\text { Fixed } \\
\text { cost }\end{array}$ & $\begin{array}{c}\text { Variable } \\
\text { cost }\end{array}$ \\
\hline 1 & 15 & 2 & 1.5 & 15 & 2.1 & 1.8 & 15 & 3 & 2.5 & 15 & 4 & 3 \\
\hline 2 & 15 & 2 & 1.5 & 15 & 2.1 & 1.8 & 15 & 3 & 2.5 & 15 & 4 & 3 \\
\hline 3 & 15 & 2 & 1.5 & 15 & 2.1 & 1.8 & 15 & 3 & 2.5 & 15 & 4 & 3 \\
\hline 4 & 15 & 2 & 1.5 & 15 & 2.1 & 1.8 & 15 & 3 & 2.5 & 15 & 4 & 3 \\
\hline 5 & 15 & 2 & 1.5 & 15 & 2.1 & 1.8 & 15 & 3 & 2.5 & 15 & 4 & 3 \\
\hline$\vdots$ & $\vdots$ & $\vdots$ & $\vdots$ & $\vdots$ & $\vdots$ & $\vdots$ & $\vdots$ & $\vdots$ & $\vdots$ & $\vdots$ & $\vdots$ & $\vdots$ \\
\hline 44 & 13.5 & 2.2 & 1.8 & 13.5 & 2.5 & 2 & 13.5 & 3 & 2.6 & 13.5 & 4.5 & 3.2 \\
\hline 45 & 18 & 2.2 & 1.8 & 18 & 2.5 & 2 & 18 & 3 & 2.6 & 18 & 4.5 & 3.2 \\
\hline 46 & 18 & 2.2 & 1.8 & 18 & 2.5 & 2 & 18 & 3 & 2.6 & 18 & 4.5 & 3.2 \\
\hline 47 & 13.5 & 2.2 & 1.8 & 13.5 & 2.5 & 2 & 13.5 & 3 & 2.6 & 13.5 & 4.5 & 3.2 \\
\hline 48 & 13.5 & 2.2 & 1.8 & 13.5 & 2.5 & 2 & 13.5 & 3 & 2.6 & 13.5 & 4.5 & 3.2 \\
\hline 49 & 18 & 2.2 & 1.8 & 18 & 2.5 & 2 & 18 & 3 & 2.6 & 18 & 4.5 & 3.2 \\
\hline 50 & 18 & 2.2 & 1.8 & 18 & 2.5 & 2 & 18 & 3 & 2.6 & 18 & 4.5 & 3.2 \\
\hline
\end{tabular}

results are no longer listed in the paper because it is not the emphasised part of this paper. The simulation example flight information is listed in Table 2, which includes the model code and flight area code. And a part of the data is listed because the data is too much. In Table 2, the last one column data is the calculating result of the model and algorithm that is, the data is the model number assigned for each flight. The data in Table 3 is the revenue and cost of each model performing each flight according to the historical data on an airline statistics. The adaptation value trace curve of model and algorithm is drawn in Figure 1.

According to the algorithm results, the model and algorithm can quickly select execution models for flights and meet the requirements of flight operation. At the same time, it is the good result. According to the chart and tables of the operation results, the improved hybrid genetic algorithm established in this paper is better than the basic genetic algorithm. The result of the operation is better, and the algorithm can quickly jump out of local optimal value. As can be seen from Figure 1, with the increase in the number of iterations, the optimization effect becomes more and more evident, but the calculating time is more and more long too. So, a specific number of iterations are needed to decide according to the actual production.

Many domestic airlines adopt the manner of manpower or half-manpower to work out FAP plan now. Considering 


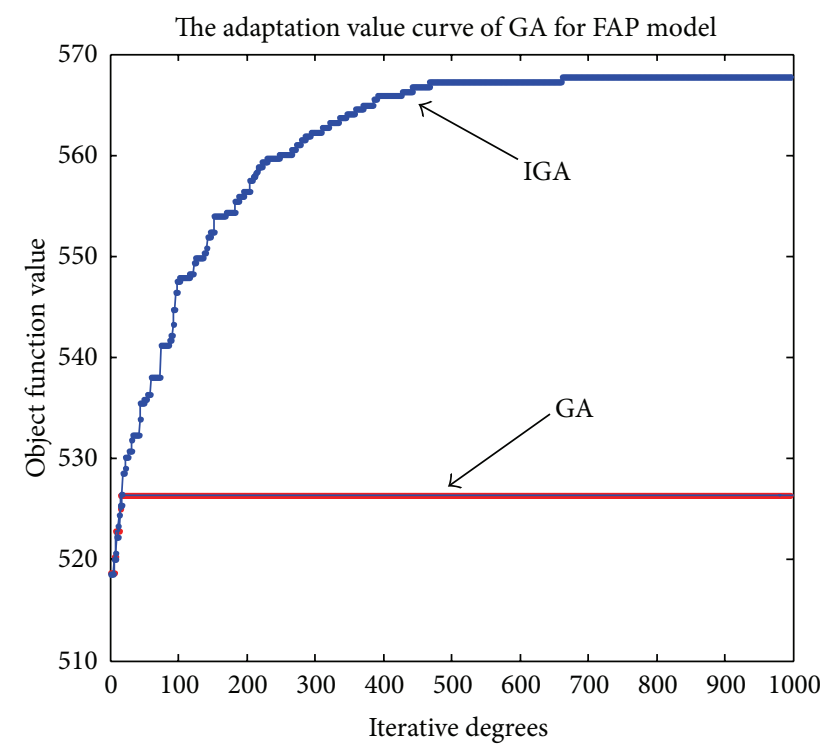

FIGURE 1: Adaptation value curve of two type algorithms (IGA and GA).

flights listed in tables, it usually takes several hours for dispatcher to weave a feasible fleet assigning plan. And the dispatcher does not have ability to consider too many flights. But with the model and algorithm proposed in this paper, it only takes no more than 1 minute to work out a fleet assigning plan, and this plan is more excellent than former. This can improve the work efficiency and save manpower resource. With the increase of the number of model types and aircraft, the number of flights increasing, that FAP plan worked out by manpower or half-manpower will become more and more difficult. However, the model and algorithm can work out the FAP plan quickly and greatly improve the level of automation.

\section{Conclusion}

In this paper, FAP in airline production scheduling is studied, and the optimization model of FAP is suggested, which considers the requirements of flight operation and takes the consolidated income maximization as the goal considering all flights. At the same time, an adaptive genetic algorithm is constructed to solve the model, which can find out the suitable solution rapidly. The researching on practical production data shows that the model and algorithm are practical and the effect of FAP planning is nice. And if this technique is applied in production scheduling and planning of airlines, the automation level of airlines will be improved, and the running cost will be reduced.

\section{Acknowledgment}

This work is supported by the Unite Foundation of National Natural Sciences Foundation of China and Civil Aviation Administration of China (no. U1233107).

\section{References}

[1] H. D. Sherali, E. K. Bish, and X. Zhu, "Airline fleet assignment concepts, models, and algorithms," European Journal of Operational Research, vol. 172, no. 1, pp. 1-30, 2006.

[2] C. Jeenanunta, B. Kasemsontitum, and T. Noichawee, "A multicommodity flow approach for aircraft routing and maintenance problem," in Proceedings of the IEEE International Conference on Quality and Reliability (ICQR '11), pp. 150-155, 2011.

[3] W. Zhang, M. Kamgarpour, D. Sun et al., "A hierarchical flight planning framework for air traffic management," Proceedings of the IEEE, vol. 100, no. 1, pp. 179-194, 2012.

[4] X. Zhu, J. Zhu, and Q. Gao, "The research on robust fleet assignment problem based on flight purity," Forecasting, vol. 30, no. 1, pp. 71-74, 2011.

[5] D. Mou and Z. Zhang, "Robust fleet scheduling problem based on probability of flight delay," Journal Of Civil Aviation University Of China, vol. 28, no. 6, pp. 35-39, 2010.

[6] K. Zhou and H. Xia, "Optimization model and algorithm for aircraft scheduling problem based on cooperative mult-task assignment," Acta Aeronautica et Astronautica Sinica, vol. 32, no. 12, pp. 2293-2301, 2011.

[7] Y. Li and T. Na, "Study on flight-string optimization based on partheno-genetic algorithm," in Proceedings of the 8th World Congress on Intelligent Control and Automation (WCICA '10), pp. 4093-4096, Jinan, China, July 2010.

[8] W. Wu, "Improved Genetic Algorithm-IGA," Computer Knowledge and Technology, vol. 8, no. 1, pp. 123-125, 2012. 


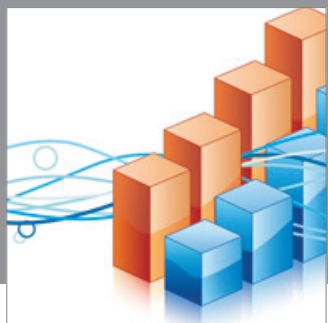

Advances in

Operations Research

mansans

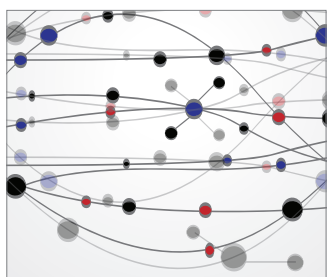

The Scientific World Journal
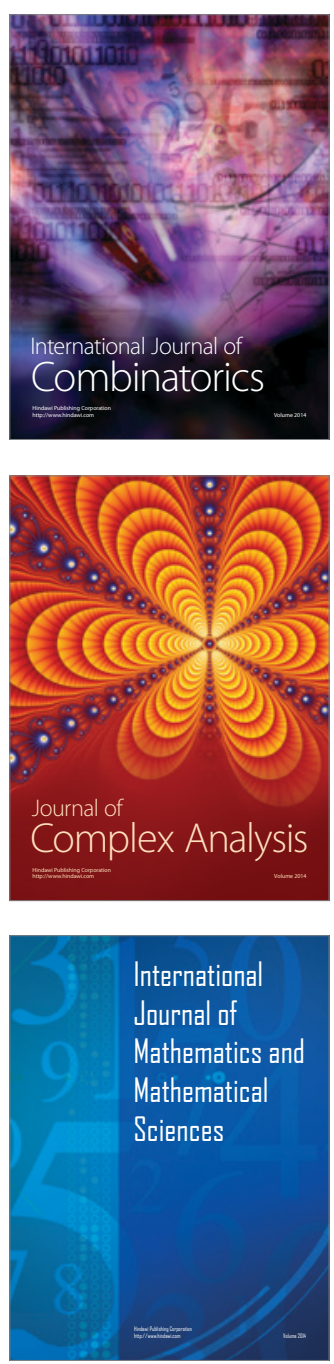
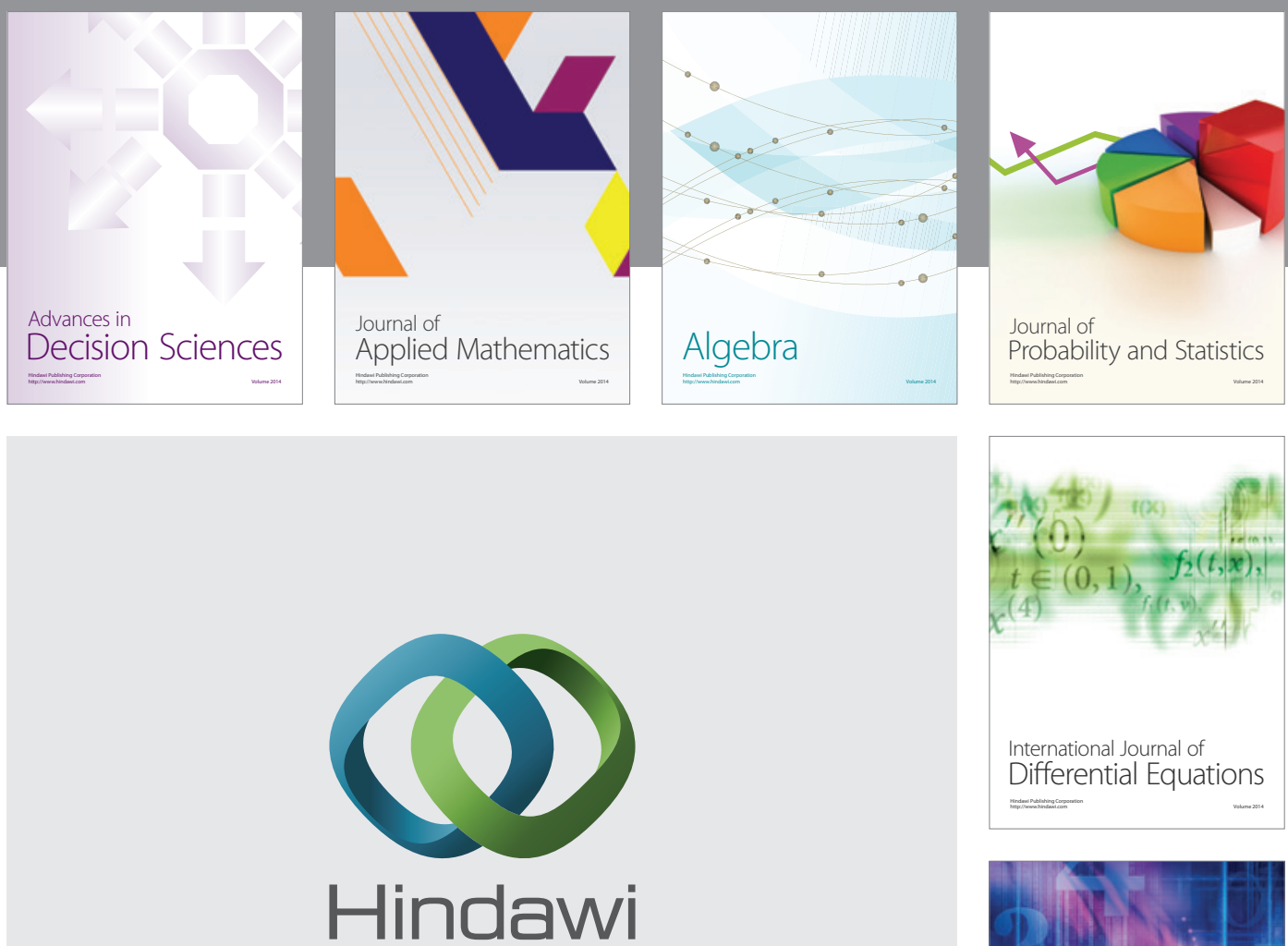

Submit your manuscripts at http://www.hindawi.com
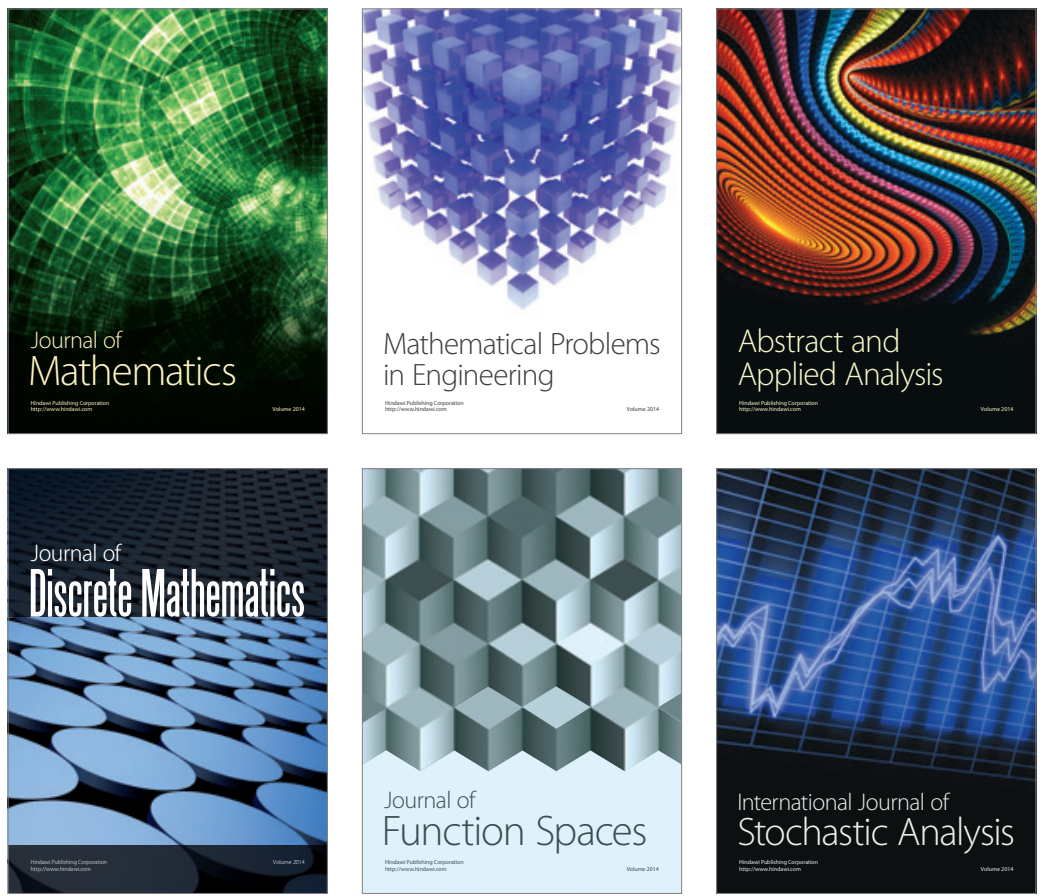

Journal of

Function Spaces

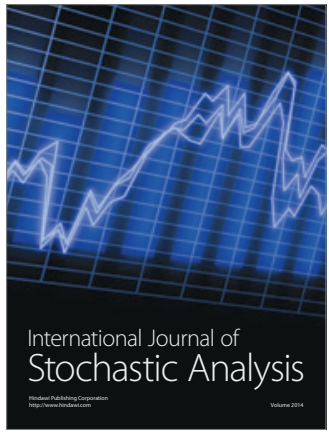

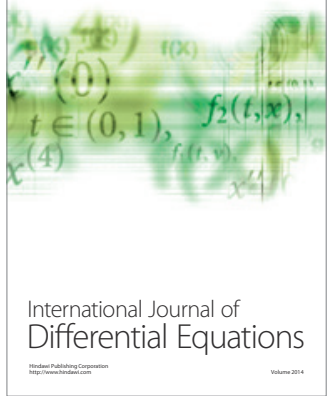
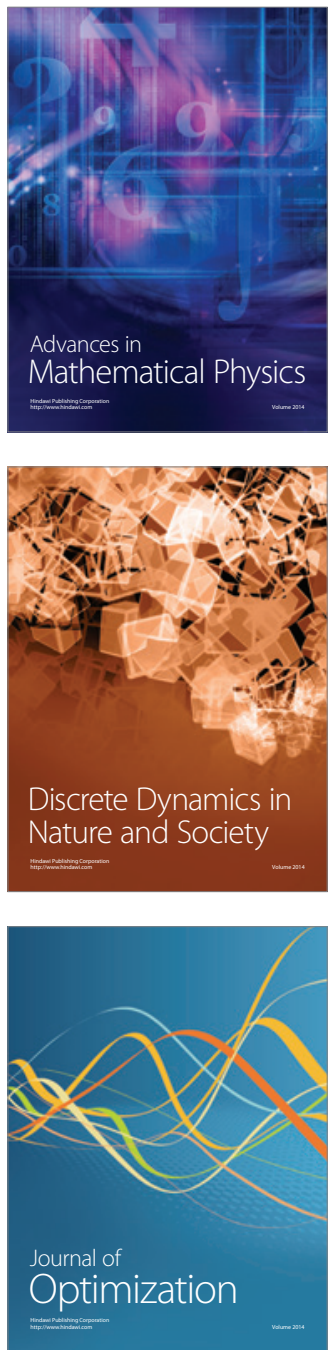